\title{
Percepción de riesgo ante el coronavirus en la primera fase de la pandemia en Argentina
}

\author{
Lorena Saletti-Cuesta* \\ Natalia Tumas** \\ Silvina Berra***
}

Recibido en 10 de agosto de 2020, aceptado en 31 de octubre de 2020

\section{Citar este artículo así:}

Saletti-Cuesta L, Tumas N, Berra S. Percepción de riesgo ante el coronavirus en la primera fase de la pandemia en Argentina. Hacia. Promoc. Salud. 2021; 26 (1): 156-171 https://doi.org/10.17151/hpsal.2021.26.1.13

\begin{abstract}
Objetivo. Analizar los factores asociados a la percepción de riesgo de coronavirus en la primera fase de la pandemia en Argentina. Métodos. Se desarrolló un estudio transversal en población adulta (n=992) argentina. En marzo 2020 se aplicó un cuestionario online de la Organización Mundial de la Salud adaptado a Argentina. Se estimó la asociación entre la percepción de probabilidad de contagio y de severidad en caso de contagio, y sentimientos en relación al coronavirus, autoeficacia y búsqueda de información, mediante modelos de regresión lineal múltiple. Resultados. La percepción de riesgo frente al coronavirus en la primera fase de la pandemia fue moderada. Sin embargo, la proporción de personas que indicó puntuaciones altas fue mucho mayor en la percepción de severidad, comparado con la percepción de probabilidad de contagio. La percepción de probabilidad de contagio fue menor a mayor percepción de lejanía del virus y a mayor frecuencia de búsqueda de información. La percepción de severidad en caso de contagio se asoció positivamente con la frecuencia de búsqueda de información. En cambio, se asoció inversamente con la percepción de lejanía del virus y la autoeficacia. Conclusiones. Existen diferentes factores tanto emocionales como sociales asociados a la percepción de contagio y de severidad en Argentina. Esta complejidad debería considerarse en el diseño de políticas públicas para la prevención de COVID-19.
\end{abstract}

\section{Palabras claves}

Infecciones por coronavirus; pandemias; evaluación del impacto en la salud; riesgo; Argentina. (fuente: DeCS, BIREME)

\footnotetext{
*Licenciada en Psicología, Doctora en por la Universidad de Granada, España. Centro de Investigaciones y Estudios sobre Cultura y Sociedad; Consejo Nacional de Investigaciones Científicas y Técnicas y Universidad Nacional de Córdoba. Argentina. Autora de correspondencia: lorenasaletti@unc.edu.ar. (D) orcid.org/0000-0002-0989-4093. Google

** Licenciada en Nutrición, Doctora en Demografía. Centro de Investigaciones y Estudios sobre Cultura y Sociedad; Consejo Nacional de Investigaciones Científicas y Técnicas y Universidad Nacional de Córdoba. Universidad Nacional de Córdoba. Universidad Católica de Córdoba. Córdoba, Argentina. natalia.tumas@unc.edu.ar (D) orcid.org/0000-0003-4730-6624. Google

${ }_{* * *}^{*}$ Licenciada en Nutrición, Doctora en Ciencias de la Salud. Centro de Investigaciones y Estudios sobre Cultura y Sociedad; Consejo Nacional de Investigaciones Científicas y Técnicas y Universidad Nacional de Córdoba. Universidad Nacional de Córdoba. Centro de Investigación Epidemiológica y en Servicios de Salud, Escuela de Salud Pública, Universidad Nacional de Córdoba, Argentina. sberra@unc.edu.ar (DD orcid. org/0000-0003-3618-7041. Google
} 


\title{
Perception of risk facing coronavirus in the first phase of the pandemic in Argentina.
}

\begin{abstract}
Objective: to analyze the factors associated with the perception of risk of coronavirus in the first phase of the pandemic in Argentina. Methods: a cross-sectional study was carried out in the Argentine adult population $(\mathrm{n}=$ 992). An online survey of the World Health Organization adapted to Argentina was applied in March-2020. The association between the perception of probability of contagion and severity in case of contagion and feelings related to the coronavirus, self-efficacy and health, were estimated using multiple linear regression models. Results: The perception of risk facing the first phase of the pandemic was moderate. However, the proportion of people who indicated high scores in the perception of severity was much higher compared to the perception of probability of contagion. The perception of probability of contagion was lower at greater perception of remoteness of the virus and it was greater at a higher frequency of information search. The perception of severity in case of contagion was positively associated with frequency of information search. Instead, it was inversely associated with the perception of remoteness of the virus and self-efficacy. Conclusions: There are different social and emotional factors associated with the perception of contagion and severity of coronavirus in Argentina. This complexity should be considered in the design of public policies for the prevention of COVID-19.
\end{abstract}

\section{Key words}

Coronavirus infections; pandemics; health impact assessment; risk; risk assessment; Argentina, access to information (source: $M e S H, N L M$ ).

\section{Percepção de risco perante o coronavírus na primeira fose do pandemio no Argentina.}

\begin{abstract}
Resumo
Objetivo. Analisar os fatores associados à percepção de risco de coronavírus na primeira fase da pandemia na Argentina. Métodos. Desenvolveu-se um estudo transversal em população adulta (n=992) na Argentina. Em março de 2020 foi aplicado um questionario online da Organização Mundial da Saúde adaptado para a Argentina. Estimouse a associação entre a percepção de probabilidade de contágio e da severidade no caso de contágio, e sentimentos em relação ao coronavírus, auto-eficácia e busca de informação, mediante modelos de regressão linear múltipla. Resultados. A percepção de risco frente ao coronavírus na primeira fase da pandemia foi moderada. Entretanto, a proporção de pessoas que indicou pontuações altas foi muito maior na percepção de severidade, comparado com a percepção de probabilidade de contágio. A percepção de probabilidade de contágio foi menor quanto maior a percepção de distância do vírus e a maior frequência de busca de informação. A percepção de severidade no caso de contágio é associada positivamente à frequência de busca de informação; por outro lado é associada inversamente à percepção de distância do vírus e a auto-eficácia. Conclusões. Existem diferentes fatores tanto emocionais como sociais associados à percepção de contágio e de severidade na Argentina. Esta complexidade deveria ser considerada no planejamento de políticas públicas para a prevenção da COVID-19.
\end{abstract}

\section{Palavros chave}

Infecções por coronavírus; pandemias; avaliação do impacto na saúde; risco; a Argentina.(fonte: DeCS, BIREME). 


\section{Introducción}

La percepción de riesgo es un concepto muyutilizado en salud pública especialmente por su vinculación con prácticas de prevención y de protección frente a diversas enfermedades o eventos (1). Mark Conner (2) destaca cuatro modelos principales para estudiar dicha vinculación: el modelo de creencias de salud, la teoría de motivación a la protección, la teoría de acción planificada y el modelo socio-cognitivo. Todos ellos se focalizan en diversos aspectos cognitivos (pensamientos, sentimientos, etc.), siendo la percepción de riesgo, o la valoración que las personas realizan de la probabilidad de que ocurra un daño si no hay una acción que lo prevenga, el concepto principal. Sin embargo, la relación entre percepción de riesgo y la adopción de medidas de protección es compleja, puesto que son dos constructos diferentes que no siempre están relacionados (1).

Si bien no hay un acuerdo sobre cómo la percepción de riesgo debe ser evaluada $(1,3,4)$ y por lo tanto existe gran heterogeneidad en su estudio (5), se reconoce que debe ser comprendida, no solo en relación con factores individuales, sino también con factores sociales y emocionales en el marco de un contexto social, cultural y económico determinado $(6,7)$.

La reciente aparición del coronavirus SARSCoV-2, que provoca la enfermedad del COVID-19, con su rápida transmisión y números de muertes, ha llevado a que la Organización Mundial de la Salud (OMS) lo declare el 11 de marzo-2020 como pandemia (8). En Argentina, el primer brote de coronavirus se inició el 3 de marzo de 2020, con la confirmación del primer caso importado y el 7 de marzo se produjo la primera muerte por esta causa. Desde febrero, el Gobierno argentino fue aplicando una serie de medidas de contención mediante la modificación de los protocolos de vuelos que llegaban zonas afectadas, la suspensión de actividades escolares, culturales y deportivas con asistencia masiva de personas y el cierre de fronteras para población extranjera no residente. Desde el 19 de marzo-2020, el gobierno dictó el aislamiento social, preventivo y obligatorio para toda la población, en respuesta a la evolución epidemiológica del COVID-19 en nuestro contexto.

Con base en experiencias anteriores, se sabe que un virus como el nuevo coronavirus genera una amenaza colectiva donde personas tienden a sentirse con pocas posibilidades de controlar el riesgo (5). Asimismo, esta percepción del riesgo está fuertemente influenciada por la naturaleza del peligro y la forma en que se comunican los casos y decesos. Por ello es importante comprender la percepción de riesgo de la población, a lo largo de las diferentes fases de un brote, para contribuir a que las personas adopten prácticas de prevención y diseñar políticas públicas adecuadas (5,9-11).

Este trabajo forma parte de un estudio más amplio, asentado en el modelo de creencias de salud para estudiar la percepción de riesgo en relación al COVID-19. El objetivo del presente trabajo es analizar los factores asociados a la percepción de riesgo en la primera fase de la pandemia en Argentina.

\section{Materiales y método}

\section{Diseño del estudio}

Se desarrolló un estudio de corte transversal, mediante una encuesta dirigida a población adulta argentina, que se distribuyó a través de redes sociales durante un lapso de 48 horas, entre el 23 y el 25 de marzo de 2020, durante la primera semana del aislamiento social, preventivo y obligatorio. Inicialmente, se realizó una convocatoria abierta a la que respondieron 3216 personas, mayoritariamente mujeres, jóvenes y de niveles educativos altos. A fin de corregir ese defecto de autoselección, se realizó luego un muestreo aleatorio estratificado por sexo y edad, de acuerdo a la distribución censal de la población argentina. 
Así, la muestra final incluyó a un total de 992 personas de entre 18 y 74 años.

En cuanto a las consideraciones éticas, cada participante brindó su consentimiento informado antes de comenzar el cuestionario y los datos se recopilaron y analizaron de manera anónima. Al finalizar el cuestionario se brindó el enlace a la web del Ministerio de Salud de la Nación para ofrecer información nacional confiable sobre COVID-19.

\section{Variables de estudio}

Como variables dependientes se consideraron la percepción de la probabilidad de contagio de COVID-19 y la percepción del grado de severidad en caso de contagio. Ambas fueron registradas en una escala Likert de 1 a 7 puntos (desde "muy poco probable" hasta "muy probable"; y desde "nada grave" hasta "muy grave", respectivamente). También fueron categorizadas para su análisis descriptivo de la siguiente manera: baja (1 a 3 puntos), media (4 puntos), alta (5 a 7 puntos).

Como variables independientes, valoradas en una escala Likert de 1 a 7 , se incluyeron las siguientes: percepción de lejanía del virus (desde "está cerca de mí" a "está lejos de mí”, percepción sobre su diseminación rápida (desde "se propaga lentamente" a "se propaga rápidamente", percepción sobre su alta promoción en los medios (desde "está muy promocionado por los medios" hasta "no está muy promocionado en los medios", frecuencia de búsqueda de información (desde "nunca" a "muchas veces al día"), y dos variables vinculadas a la autoeficacia: conocimiento percibido para protegerse (desde "no, para nada" a "sí, muchísimo") y capacidad percibida para evitar el contagio de coronavirus (desde "extremadamente difícil" a "extremadamente fácil"). Como variables de ajuste se consideraron: edad (años), identidad de género (mujer, varón, no binario, prefiere no responder), escolaridad (secundaria completa o menos, terciario incompleto o completo o universitario incompleto, universitario completo o más), tamaño poblacional de la localidad de residencia (menos de 100000 habitantes, entre 100000 y 1000000 de habitantes, más de 1000000 de habitantes), posicionamiento político (1 a 7 , escala Likert -totalmente de izquierda a totalmente de derecha), pertenencia a grupo de riesgo frente a COVID-19 (sí, no), y salud percibida (buena/ muy buena / excelente, regular / mala).

El cuestionario empleado corresponde a una adaptación al contexto argentino del cuestionario original utilizado en el estudio COSMO ("COVID-19 Snapshot MOnitoring: Monitoring knowledge, risk perceptions, preventive behaviours, and public trust in the current coronavirus outbreak") (12), elaborado por la Oficina Regional Europea de la OMS. La adaptación consistió en su traducción al idioma español, en la incorporación de las medidas preventivas frente al COVID-19 vigentes en Argentina y de otras variables de interés como percepción de salud, ideología política, entre otras. La primera versión del cuestionario fue revisada por el equipo de investigación, que además consultó a personas expertas. Se diseñó el cuestionario en la plataforma Google Forms y una versión preliminar fue testeada mediante envío a una muestra de conveniencia de 30 personas, heterogénea en cuanto a edad y sexo. Finalmente, la versión argentina del cuestionario excluyó algunas secciones del original que no resultaron comprensibles o aceptables en la prueba piloto. (versión final: https://www.psycharchives.org/ bitstream $/ 20.500 .12034 / 2401 / 2 /$ Salud $\% 20$ y $\% 20$ coronavirus $\% 20$ T $1 \% 20$ FINAL $\% 20-\% 20$ Formularios $\% 20 \mathrm{de} \% 20$ Google.pdf).

\section{Análisis estadísticos}

Inicialmente se realizó un análisis exploratorio de las variables de estudio antes mencionadas, mediante estadísticas descriptivas. Luego se valoró si existían diferencias significativas o asociaciones entre las covariables y el grado de percepción de riesgo, mediante test $\mathrm{Chi}^{2}$ y ANOVA, según corresponda. 
Posteriormente se estimaron modelos de regresión lineal múltiple para identificar los factores asociados a la percepción de riesgo, incluyendo todas las variables que en el análisis bivariado mostraron resultados cercanos a la significación estadística y las que tenían interés según el modelo conceptual presentado. A tal fin se realizó una adaptación de la sintaxis propuesta originalmente por el grupo COSMO de la Universidad de Erfurt. Todos los análisis fueron realizados con el software Stata v14.

\section{Resultodos}

De las 992 personas que integraron la muestra, 500 fueron mujeres $(50,4 \%), 461$ hombres (46,5\%), 12 personas se identificaron como no binarias $(1,2 \%)$ y 19 prefirieron no responder por su identidad de género. Participaron personas de todas las provincias de Argentina y de la Ciudad Autónoma de Buenos Aires (5,54\%), pero fue más alta la participación de residentes en la provincia de Córdoba, desde donde se lanzó la encuesta $(64,9 \%)$. La distribución por nivel de escolaridad resultó en $14,5 \%$ de nivel secundario incompleto o menor, $49,1 \%$ de terciario o universitario incompleto y $48,0 \%$ de universitario completo de grado o mayor.

\section{Percepción de riesgo de contagio}

En general, la puntuación media obtenida de la escala de 7 puntos fue 3,69 (DS=1,61). La Figura 1 muestra que el 43,6\% de la muestra percibió la probabilidad de contagio como baja en y que este porcentaje llega al $56,7 \%$ en personas de más de 60 años $(p<0,05)$.

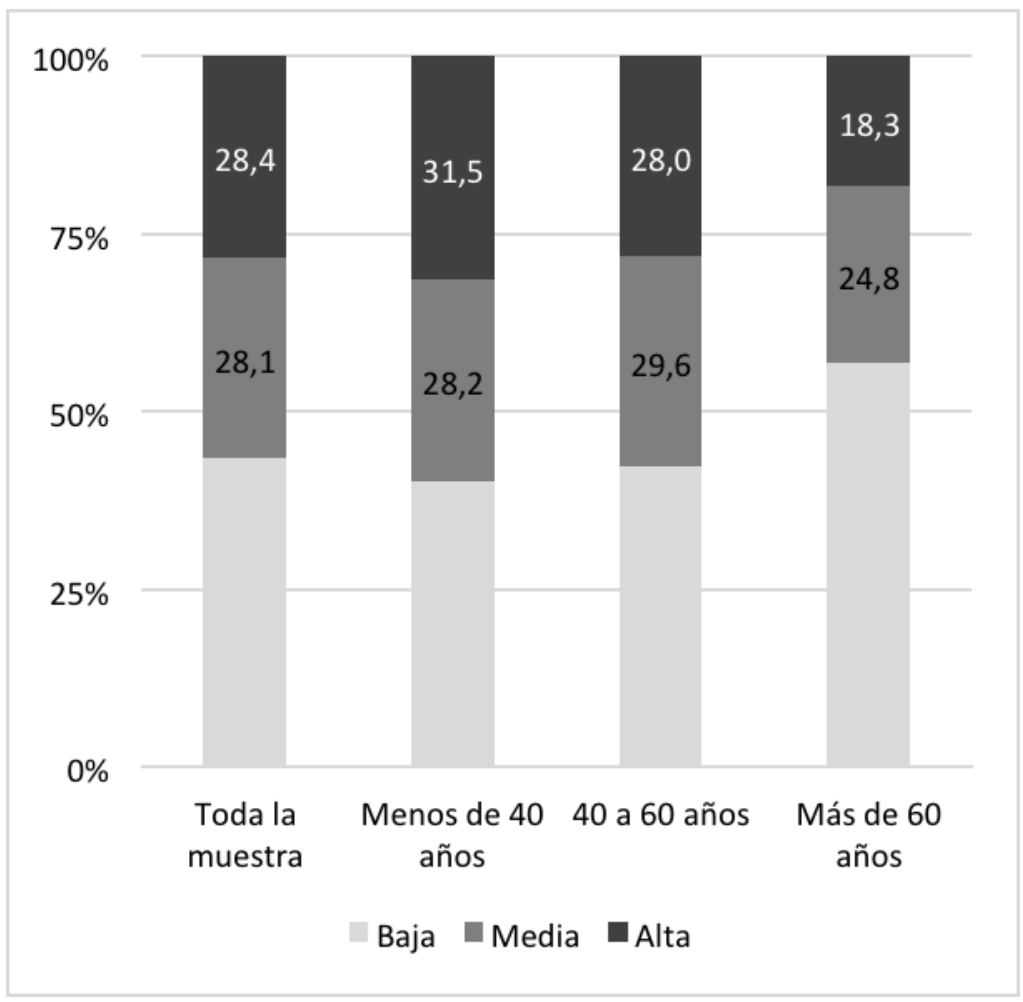

Figura 1. Porcentajes percepción de probabilidad de contagio de COVID-19 en toda la muestra y por grupos de edad. 
Las personas que tuvieron una percepción de riesgo alta (5 a 7 puntos en la escala de respuesta) tuvieron en promedio menor percepción de lejanía del virus, mayor percepción de la rapidez de diseminación del virus, mayor frecuencia de búsqueda de información y una menor sensación de facilidad para evitar el contagio $(p<0,05)$ (tabla 1). La sensación de saber cómo protegerse fue levemente menor en quienes tuvieron una percepción de riesgo media en comparación con quienes tuvieron una percepción de riesgo baja o alta. Además, entre las personas que tuvieron una percepción de riesgo baja, tanto el promedio de edad como el posicionamiento de ideas políticas hacia la derecha fueron mayores $(\mathrm{p}<0,05)$.

Tabla 1. Características de las personas según probabilidad de contagio percibida.

\begin{tabular}{|c|c|c|c|c|c|c|c|}
\hline \multirow{3}{*}{ Características de las personas } & \multicolumn{7}{|c|}{ Probabilidad de contagio percibida } \\
\hline & \multirow{2}{*}{$\begin{array}{c}\text { Total } \\
n(\%)\end{array}$} & \multicolumn{2}{|c|}{ Baja } & \multicolumn{2}{|c|}{ Media } & \multicolumn{2}{|c|}{ Alta } \\
\hline & & $n(\%)$ & $m($ d.e. $)$ & $n(\%)$ & $m($ d.e. $)$ & $n(\%)$ & $m($ d.e. $)$ \\
\hline $\begin{array}{l}\text { Percepción de lejanía del virus, escala } \\
1-7 *\end{array}$ & 989 & 432 & $4,9(1,5)$ & 277 & $4,2(1,3)$ & 280 & $3,6(1,4)$ \\
\hline $\begin{array}{l}\text { Percepción sobre diseminación rápida } \\
\text { del virus, escala } 1-7 *\end{array}$ & 991 & 432 & $5,9(1,5)$ & 278 & $5,8(1,5)$ & 281 & $6,2(1,1)$ \\
\hline $\begin{array}{l}\text { Percepción sobre la promoción en los } \\
\text { medios, escala } 1-7\end{array}$ & 986 & 430 & $2,2(1,4)$ & 277 & $2,3(1,4)$ & 279 & $2,2(1,4)$ \\
\hline $\begin{array}{l}\text { Frecuencia de búsqueda de información, } \\
\text { escala } 1-7^{*}\end{array}$ & 992 & 432 & $5,3(1,5)$ & 279 & $5,5(1,4)$ & 281 & $5,6(1,4)$ \\
\hline $\begin{array}{l}\text { Conocimiento percibido sobre } \\
\text { protección, escala } 1-7^{*}\end{array}$ & 990 & 431 & $5,6(1,1)$ & 279 & $5,4(1,1)$ & 280 & $5,6(1,0)$ \\
\hline $\begin{array}{l}\text { Capacidad percibida para evitar contagio, } \\
\text { escala } 1-7 *\end{array}$ & 988 & 430 & $4,8(1,5)$ & 279 & $4,6(1,2)$ & 279 & $4,5(1,3)$ \\
\hline $\begin{array}{l}\text { Posicionamiento político izquierda/ } \\
\text { derecha, escala } 1-7^{*}\end{array}$ & 976 & 424 & $3,8(1,4)$ & 274 & $3,5(1,3)$ & 278 & $3,6(1,3)$ \\
\hline Edad* & $\begin{array}{c}992 \\
(100)\end{array}$ & $432(43,6)$ & $\begin{array}{c}42,7 \\
(17,3)\end{array}$ & $\begin{array}{c}279 \\
(28,1) \\
\end{array}$ & $\begin{array}{c}41,2 \\
(15,2)\end{array}$ & $\begin{array}{c}281 \\
(28,4) \\
\end{array}$ & $39,4(14,3)$ \\
\hline $\begin{array}{l}\text { Identidad de género } \\
\text { Hombre } \\
\text { Mujer } \\
\text { No binario } \\
\text { Prefiere no decir }\end{array}$ & $\begin{array}{c}455 \\
(45,9) \\
507 \\
(51,1) \\
12(1,2) \\
18(1,8)\end{array}$ & $\begin{array}{c}199(46,1) \\
222(51,4) \\
6(1,4) \\
5(1,2)\end{array}$ & $\begin{array}{l}- \\
- \\
- \\
-\end{array}$ & $\begin{array}{c}121 \\
(43,4) \\
146 \\
(52,3) \\
3(1,1) \\
9(3,2)\end{array}$ & $\begin{array}{l}- \\
- \\
-\end{array}$ & $\begin{array}{c}135 \\
(48,0) \\
139 \\
(49,5) \\
3(1,1) \\
4(1,4)\end{array}$ & $\begin{array}{l}- \\
- \\
- \\
-\end{array}$ \\
\hline
\end{tabular}




\begin{tabular}{|c|c|c|c|c|c|c|c|}
\hline \multirow{2}{*}{ Características de las personas } & \multicolumn{7}{|c|}{ Probabilidad de contagio percibida } \\
\hline & Total & Baja & & & & & \\
\hline $\begin{array}{l}\text { Escolaridad } \\
\text { Secundario completo o menos } \\
\text { Terciario o universit. Incompleto } \\
\text { Universitario completo }\end{array}$ & $\begin{array}{c}144 \\
(14,5) \\
372 \\
(37,5) \\
476 \\
(48,0)\end{array}$ & $\begin{array}{c}69(16,0) \\
170(39,3) \\
193(44,7)\end{array}$ & $\begin{array}{l}- \\
-\end{array}$ & $\begin{array}{c}44 \\
(15,8) \\
103 \\
(36,9) \\
132 \\
(47,3)\end{array}$ & $\begin{array}{l}- \\
- \\
-\end{array}$ & $\begin{array}{c}31 \\
(11,0) \\
99 \\
(35,2) \\
151 \\
(53,8)\end{array}$ & . \\
\hline $\begin{array}{l}\text { Tamaño de la localidad de residencia } \\
>1.000 .000 \text { hab. } \\
100.000 \text { a } 1.000 .000 \text { hab. } \\
\leq 100.000 \text { hab. }\end{array}$ & $\begin{array}{c}486 \\
(49,0) \\
221 \\
(22,3) \\
285 \\
(28,7) \\
\end{array}$ & $\begin{array}{c}202(46,8) \\
96(22,2) \\
134(31,0)\end{array}$ & $\begin{array}{l}- \\
- \\
-\end{array}$ & $\begin{array}{c}141 \\
(50,5) \\
67 \\
(24,0) \\
71 \\
(25,5) \\
\end{array}$ & $\begin{array}{l}- \\
- \\
-\end{array}$ & $\begin{array}{c}143 \\
(50,9) \\
58 \\
(20,6) \\
80 \\
(28,5) \\
\end{array}$ & 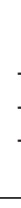 \\
\hline $\begin{array}{l}\text { Salud percibida } \\
\text { Buena, muy buena o excelente } \\
\text { Regular o mala }\end{array}$ & $\begin{array}{c}947 \\
(95,7) \\
43(4,3)\end{array}$ & $\begin{array}{c}416(96,3) \\
16(3,7)\end{array}$ & - & $\begin{array}{c}267 \\
(94,3) \\
11(5,7)\end{array}$ & - & $\begin{array}{c}264 \\
(94,3) \\
16(5,7)\end{array}$ & . \\
\hline $\begin{array}{l}\text { Pertenencia a grupo de riesgo } \\
\text { Sí } \\
\text { No }\end{array}$ & $\begin{array}{c}257 \\
(28,1) \\
656 \\
(71,9)\end{array}$ & $\begin{array}{l}121(30,0) \\
282(70,0)\end{array}$ & - & $\begin{array}{l}73 \\
(29,1) \\
178 \\
(70,9)\end{array}$ & - & $\begin{array}{c}63 \\
(24,3) \\
196 \\
(75,7)\end{array}$ & . \\
\hline
\end{tabular}

Categorías de percepción de probabilidad de contagio: baja (1 a 3 puntos), media (4 puntos), alta (5 a 7 puntos); $m$ (d.e.): media (desvío estándar). \%: Porcentaje.

$* p<0,05$ prueba ANOVA para variables cuantitativas

$\dagger \mathrm{p}<0,05$ prueba $X^{2}$ para variables categóricas

Fuente: Elaboración propia.

En el análisis de las características personales asociadas a la percepción de riesgo de contagio mediante regresión lineal multivariada (tabla 2), muestra una mayor lejanía del virus (beta $=-0,38$; intervalo de confianza del 95 (IC95\%)=-0,45 $-0,31)$, y una mayor frecuencia de búsqueda de información (beta $=0,09 ;$ IC95\% $=0,02-0,16$ ) se asocian a una menor percepción de riesgo de contagio. En cuanto a las variables de ajuste, la percepción de riesgo de contagio fue de menor a mayor edad (beta $=-0,02$; IC95\%=-0,02 - -0,01), en las de menor nivel de escolaridad en comparación con las de nivel universitario completo o más (beta $=-0,36$; IC95\%=-0,67 - -0,05) y en la medida en que manifestaban que sus ideas políticas tendían hacia la derecha (beta $=-0,10$; IC95\%=$0,17--0,02)$. 
Tabla 2. Medidas de asociación entre las características de las personas y la probabilidad de contagio de COVID-19 percibida.

\begin{tabular}{|c|c|c|}
\hline \multirow{2}{*}{ Variables } & \multicolumn{2}{|c|}{ Probabilidad de contagio percibida $(n=844)$} \\
\hline & $b(I C 95 \%)$ & valor $p$ \\
\hline Percepción de lejanía del virus escala 1-7 & $-0,38(-0,45--0,31)$ & $<0,001$ \\
\hline Percepción sobre diseminación rápida del virus escala 1-7 & $0,02(-0,05-0,09)$ & 0,548 \\
\hline Percepción sobre la promoción en los medios escala 1-7 & $0,01(-0,06-0,08)$ & 0,842 \\
\hline Frecuencia de búsqueda de información escala 1-7 & $0,09(0,02-0,16)$ & 0,011 \\
\hline Conocimiento percibido sobre protección escala 1-7 & $-0,01(-0,11-0,81)$ & 0,790 \\
\hline Capacidad percibida para evitar contagio escala 1-7 & $-0,03(-0,10-0,04)$ & 0,412 \\
\hline Edad & $-0,02(-0,02--0,01)$ & $<0,001$ \\
\hline \multicolumn{3}{|l|}{ Identidad de género: } \\
\hline Hombre & Ref. & - \\
\hline Mujer & $0,04(-0,16-0,25)$ & 0,682 \\
\hline No binario & $-0,53(-1,40-0,33)$ & 0,226 \\
\hline Prefiere no decir & $0,04(-0,80-0,88)$ & 0,927 \\
\hline \multicolumn{3}{|l|}{ Escolaridad: } \\
\hline Universitario completo & Ref. & - \\
\hline Terciario o universit. incompleto & $-0,36(-0,67-0,05)$ & 0,022 \\
\hline Secundario completo o menos & $-0,17(-0,39-0,56)$ & 0,143 \\
\hline \multicolumn{3}{|l|}{ Tamaño de la localidad de residencia: } \\
\hline$>1.000 .000$ hab. & Ref. & - \\
\hline 100.000 a 1.000 .000 hab. & $0,15(-0,10-0,41)$ & 0,240 \\
\hline$\leq 100.000$ hab. & $0,06(-0,17-0,30)$ & 0,600 \\
\hline \multicolumn{3}{|l|}{ Salud percibida: } \\
\hline Buena, muy buena o excelente & Ref. & - \\
\hline Regular o mala & $0,04(-0,47-0,56)$ & 0,868 \\
\hline \multicolumn{3}{|l|}{ Pertenencia a grupo de riesgo: } \\
\hline No & Ref. & - \\
\hline Sí & $0,06(-0,21-0,33)$ & 0,660 \\
\hline \multicolumn{3}{|l|}{ Posicionamiento político izquierda/ derecha escala 1-7 } \\
\hline & $-0,10(-0,17--0,02)$ & 0,014 \\
\hline
\end{tabular}

Fuente: Elaboración propia. 


\section{Percepción de severidad}

En términos generales, la puntuación media obtenida de la escala de 7 puntos para la percepción de severidad fue de 4,55 (DS=1,72). La distribución porcentual en categorías de la percepción de severidad se muestra en la Figura
2. Mientras que el 48,9\% de la muestra manifestó una severidad percibida alta, la comparación por grupos de edades muestra cómo esta percepción aumenta con la edad, con un $80,4 \%$ de las personas mayores de 60 años ubicándose en esa categoría (figura 2).

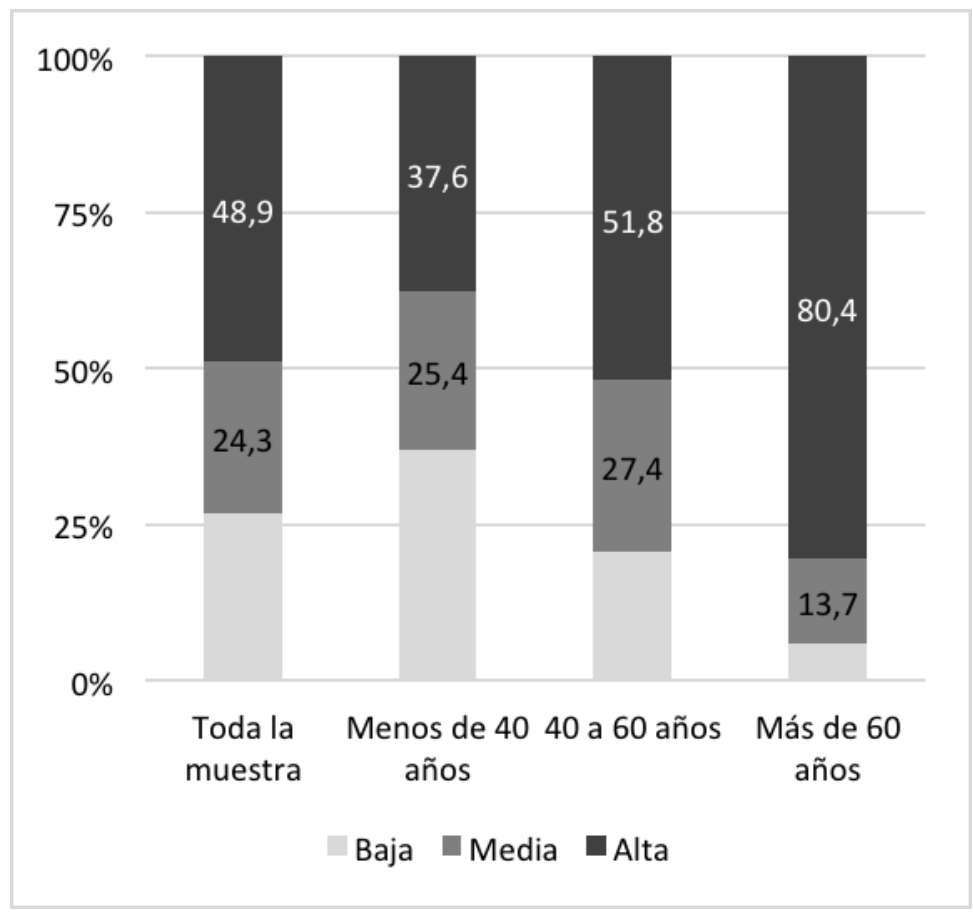

Figura 2. Porcentajes percepción de severidad en caso de contagio de COVID-19 en toda la muestra y por grupos de edad.

Fuente: Elaboración propia.

Adicionalmente, las personas con una alta percepción de severidad tuvieron promedios más altos de percepción sobre diseminación rápida, de promoción de la situación en los medios y de frecuencia con la que se informa, mientras que puntuaron más bajo en su percepción de lejanía del virus y de facilidad para evitar el contagio $(p<0,05)$. La declaración de saber cómo protegerse frente al coronavirus fue mayor en las categorías extremas de severidad percibida $(\mathrm{p}<0,05)$ (tabla 3$)$. Además, entre quienes tuvieron una percepción de severidad alta, el promedio de edad fue mayor (46,3 años); y hubo un porcentaje evidentemente mayor de personas que declararon su salud percibida como regular o mala $(7,4 \%)$ y que manifestaron pertenecer a un grupo de riesgo $(48,3 \%)(\mathrm{p}<0,05)$. 
Tabla 3. Características de las personas según severidad percibida.

\begin{tabular}{|c|c|c|c|c|c|c|c|}
\hline \multirow{3}{*}{$\begin{array}{l}\text { Características de las } \\
\text { personas }\end{array}$} & \multicolumn{7}{|c|}{ Severidad percibida } \\
\hline & \multirow{2}{*}{$\begin{array}{l}\text { Total } \\
n(\%)\end{array}$} & \multicolumn{2}{|c|}{ Baja } & \multicolumn{2}{|c|}{ Media } & \multicolumn{2}{|c|}{ Alta } \\
\hline & & $n(\%)$ & $m$ (d.e.) & $n(\%)$ & $m$ (d.e.) & $n(\%)$ & $m$ (d.e.) \\
\hline $\begin{array}{l}\text { Percepción de lejanía del } \\
\text { virus, escala } 1-7^{*}\end{array}$ & 989 & 266 & $4,74,7(1,5)$ & 240 & $4,3(1,4)$ & 483 & $4,2(1,5)$ \\
\hline $\begin{array}{l}\text { Percepción sobre } \\
\text { diseminación rápida del } \\
\text { virus, escala } 1-7 *\end{array}$ & 991 & 266 & $5,7(1,5)$ & 240 & $6,0(1,34)$ & 485 & $6,1(1,3)$ \\
\hline $\begin{array}{l}\text { Percepción sobre la } \\
\text { promoción en los medios, } \\
\text { escala } 1-7 *\end{array}$ & 986 & 265 & $2,1(1,4)$ & 239 & $2,1(1,3)$ & 482 & $2,3(1,5)$ \\
\hline $\begin{array}{l}\text { Frecuencia de búsqueda de } \\
\text { información, escala } 1-7^{*}\end{array}$ & 992 & 266 & $5,1(1,6)$ & 241 & $5,3(1,4)$ & 485 & $5,7(1,4)$ \\
\hline $\begin{array}{l}\text { Conocimiento percibido } \\
\text { sobre protección, escala } \\
1-7 *\end{array}$ & 990 & 265 & $5,7(1,1)$ & 241 & $5,3(1,1)$ & 484 & $5,6(1,1)$ \\
\hline $\begin{array}{l}\text { Capacidad percibida para } \\
\text { evitar contagio, escala } 1-7 *\end{array}$ & 988 & 264 & $5,0(1,4)$ & 241 & $4,7(1,3)$ & 483 & $4,5(1,4)$ \\
\hline $\begin{array}{l}\text { Posicionamiento político } \\
\text { izquierda/ derecha, escala } \\
1-7\end{array}$ & 976 & 262 & $3,7(1,4)$ & 236 & $3,7(1,3)$ & 478 & $3,6(1,3)$ \\
\hline Edad* & $992(100)$ & $266(26,8)$ & $34,1(12,1)$ & $241(24,3)$ & $39,4(14,1)$ & $485(48,9)$ & $46,3(16,9)$ \\
\hline \multicolumn{8}{|l|}{ Identidad de género } \\
\hline Hombre & $455(45,9)$ & $137(51,5)$ & - & $106(44,0)$ & - & $212(43,7)$ & - \\
\hline Mujer & $507(51,1)$ & $122(45,9)$ & - & $124(51,4)$ & - & $261(53,8)$ & - \\
\hline No binario & $12(1,2)$ & $3(1,1)$ & - & $4(1,7)$ & - & $5(1,0)$ & - \\
\hline Prefiere no decir & $18(1,8)$ & $4(1,5)$ & - & $7(2,9)$ & - & $7(1,5)$ & - \\
\hline \multicolumn{8}{|l|}{ Escolaridad } \\
\hline Secundario completo o & $144(14,5)$ & $36(13,5)$ & - & $32(13,3)$ & - & $76(15,6)$ & - \\
\hline menos & $372(37,5)$ & $93(35,0)$ & - & $93(38,6)$ & - & $186(38,4)$ & - \\
\hline Terciario o universit. & $476(48,0)$ & $137(51,5)$ & - & $116(48,1)$ & - & $223(46,0)$ & - \\
\hline \multicolumn{2}{|l|}{ Universitario completo } & & & & & & \\
\hline $\begin{array}{l}\text { Tamaño de la localidad de } \\
\text { residencia }\end{array}$ & \multicolumn{7}{|c|}{ Tamaño de la localidad de } \\
\hline$>1.000 .000$ hab. & $221(22,3)$ & $62(23,3)$ & - & $46(19,1)$ & - & $113(23,3)$ & - \\
\hline $\begin{array}{l}100.000 \text { a } 1.000 .000 \text { hab. } \\
\leq 100.000 \text { hab. }\end{array}$ & $285(28,7)$ & $62(23,3)$ & - & $78(32,4)$ & - & $145(29,9)$ & - \\
\hline \multicolumn{8}{|l|}{ Salud percibida $\dagger$} \\
\hline Buena, muy buena o & $947(95,7)$ & $262(98,5)$ & - & $237(98,7)$ & - & $448(92,6)$ & - \\
\hline $\begin{array}{l}\text { excelente } \\
\text { Regular o mala }\end{array}$ & $43(4,3)$ & $4(1,5)$ & - & $3(1,3)$ & - & $36(7,4)$ & - \\
\hline \multicolumn{6}{|l|}{ Pertenencia a grupo de } & $210(48,3)$ & - \\
\hline $\begin{array}{l}\text { Sí } \\
\text { No }\end{array}$ & $656(71,9)$ & $246(95,3)$ & - & $185(84,1)$ & - & $225(51,7)$ & - \\
\hline & $\begin{array}{l}\text { Categorías } \\
\text { media (des } \\
{ }^{*} \mathrm{p}<0,05 \mathrm{pr} \\
\dagger \mathrm{p}<0,05 \mathrm{pr}\end{array}$ & $\begin{array}{l}\text { percepción d } \\
\text { estándar). \% } \\
\text { a ANOVA pa } \\
\text { oa } X^{2} \text { para va }\end{array}$ & $\begin{array}{l}\text { everidad: baja } \\
\text { orcentaje. } \\
\text { variables cua } \\
\text { bles categóric }\end{array}$ & $\begin{array}{l}\text { a } 3 \text { puntos), } \\
\text { tativas }\end{array}$ & dia (4 puntos & alta $(5$ a 7 pu & os); m (d.e.): \\
\hline
\end{tabular}

Fuente: Elaboración propia. 
En el análisis multivariado, la severidad percibida (tabla 4) se asoció positivamente con la frecuencia de búsqueda de información (beta $0,12$; IC $95 \%=0,04-0,19)$. Por el contrario, la percepción de severidad presentó una relación inversa con la percepción de lejanía del virus (beta-0,13; IC95\%=-0,20- 0,06 ) y con la facilidad para evitar el contagio (beta-0,11; IC95\%=-0,18
- -0,03). En cuanto a las variables de ajuste, la severidad percibida se asoció positivamente con la edad (beta $=0,19 ;$ IC95\% $=0,01-0,03$ ), la identidad de género mujer (beta $=0,26$; IC $95 \%=0,06-0,47$ ), las categorías más bajas de escolaridad (beta $=0,26$; IC95\% $=0,40-0,48)$, y la pertenencia a un grupo de riesgo frente al COVID-19 (beta $=1,28$; IC95\%=1,01 - 1,54).

Tabla 4. Medidas de asociación entre las características de las personas y la severidad percibida en caso de contagio de COVID-19.

\begin{tabular}{|c|c|c|}
\hline \multirow[t]{2}{*}{ Variables } & \multicolumn{2}{|c|}{$\begin{array}{l}\text { Severidad percibida } \\
\qquad(\mathrm{n}=\mathbf{8 4 2})\end{array}$} \\
\hline & b (IC 95\%) & valor $\mathbf{p}$ \\
\hline Percepción de lejanía del virus escala 1-7 & $-0,13(-0,20--0,06)$ & $<0,001$ \\
\hline Percepción sobre diseminación rápida del virus escala 1-7 & $0,06(-0,01-0,14)$ & 0,078 \\
\hline Percepción sobre la promoción en los medios escala 1-7 & $0,02(-0,05-0,09)$ & 0,535 \\
\hline Frecuencia de búsqueda de información escala 1-7 & $0,12(0,04-0,19)$ & 0,001 \\
\hline Conocimiento percibido sobre protección escala 1-7 & $-0,09(-0,18-0,01)$ & 0,079 \\
\hline Capacidad percibida para evitar contagio escala 1-7 & $-0,11(-0,18--0,31)$ & 0,004 \\
\hline Edad & $0,19(0,01-0,03)$ & $<0,001$ \\
\hline $\begin{array}{l}\text { Identidad de género: } \\
\text { Hombre } \\
\text { Mujer } \\
\text { No binario } \\
\text { Prefiere no decir }\end{array}$ & $\begin{array}{c}\text { Ref. } \\
0,26(0,06-0,47) \\
0,40(-0,47-1,26) \\
0,30(-0,54-1,39) \\
\end{array}$ & $\begin{array}{c}- \\
0,012 \\
0,367 \\
0,488 \\
\end{array}$ \\
\hline $\begin{array}{l}\text { Escolaridad: } \\
\text { Universitario completo } \\
\text { Terciario o universit. incompleto } \\
\text { Secundario completo o menos } \\
\end{array}$ & $\begin{array}{c}\text { Ref. } \\
0,64(0,33-0,95) \\
0,26(0,40-0,48) \\
\end{array}$ & $\begin{array}{c}- \\
<0,001 \\
0,021 \\
\end{array}$ \\
\hline $\begin{array}{l}\text { Tamaño de la localidad de residencia: } \\
>1.000 .000 \text { hab. } \\
100.000 \text { a } 1.000 .000 \text { hab. } \\
100.000 \text { a } 1.00\end{array}$ & $\begin{array}{c}\text { Ref. } \\
0,19(-0,13-0,38) \\
0,07(-0,16-0,31) \\
\end{array}$ & $\begin{array}{c}- \\
0,324 \\
0,527 \\
\end{array}$ \\
\hline $\begin{array}{l}\text { Salud percibida: } \\
\text { Buena, muy buena o excelente } \\
\text { Regular o mala }\end{array}$ & $\begin{array}{c}\text { Ref. } \\
0,38(-0,13-0,90)\end{array}$ & 0,144 \\
\hline $\begin{array}{l}\text { Pertenencia a grupo de riesgo: } \\
\text { No } \\
\text { Sí }\end{array}$ & $\begin{array}{c}\text { Ref. } \\
1,28(1,01-1,54) \\
\end{array}$ & $\begin{array}{c}- \\
<0,001 \\
\end{array}$ \\
\hline Posicionamiento político izquierda/ derecha escala 1-7 & $-0,00(-0,08-0,07)$ & 0,952 \\
\hline
\end{tabular}

Fuente: Elaboración propia. 


\section{Discusión}

El objetivo de este trabajo fue analizar los factores asociados a la percepción de riesgo en la primera fase de la pandemia en Argentina. Los resultados indicaron que la percepción de riesgo promedio entre las personas participantes fue moderada, tanto en percepción de la probabilidad de contagio como de severidad en caso de contagio. Sin embargo, la proporción de personas que indicó puntuaciones altas fue mucho mayor en la percepción de severidad comparado a la percepción de probabilidad de contagio. Además, se evidenció que factores de diversa índole mostraron asociación con tales percepciones de riesgo.

A pesar de la heterogeneidad en la definición teórica y operacional de riesgo utilizada, los resultados sobre la magnitud de la percepción de riesgo coinciden con lo observado en otros contextos en la primera fase de la pandemia (1316). Por el contrario, otros dos estudios de países asiáticos, reportan alta percepción de riesgo frente al COVID-19 entre sus participantes $(17,18)$. También un estudio comparativo (19) reporta alta percepción de riesgo en diversos países, vinculada principalmente a las experiencias directas de las personas con el virus, resaltando la importancia de los sentimientos vs. la cognición en la percepción de riesgo. La diferencia de la percepción de riesgo en Argentina respecto a, por ejemplo, los países asiáticos, podría deberse a la lejanía geográfica con el primer brote de la pandemia. Pero también, a que en el momento en que se realizó el estudio, el número de casos de contagio y muertes por COVID-19 era escaso (20) (301 casos y 4 muertes), la transmisión comunitaria era incipiente y se iniciaba recientemente la medida de aislamiento social preventivo y obligatorio. Estos factores probablemente hayan contribuido a minimizar la percepción de riesgo de contagio frente al coronavirus entre las personas participantes de nuestro estudio. Asimismo, la moderada percepción (promedio) de riesgo hallada en nuestro estudio podría deberse al sesgo optimista. En general, las personas con buena salud como la de nuestra muestra, tienen expectativas optimistas que suelen mantenerse a pesar de una realidad adversa o de recibir información sobre el riesgo de enfermarse (21).

Por otro lado, aunque la magnitud de las asociaciones fue pequeña, una mayor frecuencia de búsqueda de información se asoció tanto al aumento en la percepción de probabilidad de contagio de COVID-19 como de severidad. Esta asociación también fue identificada en otros contextos $(15,18,22)$. La influencia de la información que se recibe en la percepción de riesgo y por tanto, en la adopción de comportamientos preventivos ha sido estudiada en otras pandemias, como la generada por influenza virus H1N1 (23-25). Es por ello que los modelos de comunicación de crisis (26) recomiendan estudiar la percepción de riesgo de la población y características de la información en la que confían.

Además, en este trabajo pudo identificarse una relación inversa entre el sentimiento de lejanía del coronavirus y tanto la percepción de contagio como de severidad, a pesar de que la magnitud de las asociaciones fue escasa. La importancia de los sentimientos vinculados a la percepción de riesgo ha sido señalada en otros estudios vinculados a la COVID-19(19). Los resultados son consistentes con la hipótesis del riesgo como sentimiento, que apunta a resaltar la importancia de los factores emocionales, experimentales, en la percepción de riesgo $(27,28)$.

Por otro lado, la importancia de la autoeficacia en relación a la percepción de riesgo ha sido destacada en la literatura científica $(5,29)$. En coincidencia con otros estudios respecto al COVID-19(16,19), los resultados obtenidos señalan que una variable vinculada a la autoeficacia (capacidad percibida para evitar contagio) se asocia a la percepción de severidad, en el sentido de que a mayor autoeficacia menor percepción de severidad, 
pero no a la percepción de contagio. Cuando las personas perciben alta autoeficacia tienen mayores probabilidades de realizar comportamientos preventivos (30) lo cual podría explicar la menor percepción de severidad de la infección en caso de contagio de COVID-19 observada en nuestro estudio. La no asociación de esta variable con la percepción de probabilidad de contagio podría explicarse por la ambigüedad vinculada a las pandemias, donde hay una amenaza externa difícil de controlar, como es el virus pero que, a la vez, este agente externo depende del comportamiento cotidiano de las personas para expandirse. Estos comportamientos (lavado de manos, distancia social) son voluntarios y controlables en parte, y es por ello que son percibidos, al mismo tiempo, como poco riesgosos (5).

Además, en nuestro estudio, las variables de ajuste referidas a pertenecer a alguno de los grupos de riesgo frente a COVID-19 y edad se asociaron a una mayor percepción de severidad, como es de esperar, puesto que podrían ser personas más informadas acerca de la mayor severidad de la infección en su caso. Los resultados obtenidos indican también que a mayor edad menor percepción de probabilidad de contagio, lo que puede relacionarse con que en ese momento de la pandemia las principales personas contagiadas en nuestro país eran más jóvenes, principalmente llegadas del exterior. Según datos del Ministerio de Salud a fecha de la encuesta, el 2\% de los casos registrados correspondía a menores de 14 años, el $79 \%$ a casos a personas de entre 15 y 59 años; y el $19 \%$ a mayores de $60(20)$.

También se estudiaron otras variables sociodemográficas y socioculturales que, pudiendo estar asociadas a las percepciones de riesgo, aportan información para abordajes preventivos. Por ejemplo, reportar una ideología más cercana a la derecha se asoció, aunque con escasa magnitud, a una menor percepción de probabilidad de contagio, en coincidencia con lo identificado en Inglaterra y Estados Unidos donde una ideología conservadora se asocia a una menor percepción de riesgo (19). Sin embargo, ello no se asoció con la percepción de la severidad.

Por otro lado, se evidenció asociación entre percepción de severidad e identidad de género mujer, en coincidencia con otros estudios $(19,22,31)$. Estudios recientes indican que la prevalencia de contagio entre hombres y mujeres es similar, sin embargo, la infección es más severa en los hombres (32). La literatura indica que en, en general, las mujeres suelen reportar mayores niveles de percepción de riesgo a diversas enfermedades $(7,33)$.

En cuanto al nivel educativo, los resultados señalaron que un menor nivel educativo, se asoció una menor percepción de probabilidad de contagio y a una mayor severidad en caso de contagio. Un estudio comparativo de diversos países no encontró asociaciones entre esta variable y la percepción de riesgo frente al COVID-19 (19). Sin embargo, en Brasil el menor nivel educativo se asoció a una percepción menor de riesgo de contagio de coronavirus (31) al igual que en nuestro estudio, posiblemente por la mayor semejanza sociocultural entre Argentina y Brasil. La asociación entre el menor nivel educativo con una menor percepción de riesgo de contagio, podría llevar a una menor adhesión de medidas preventivas en estos grupos, lo cual debe tenerse muy en cuenta en el diseño de estrategias en nuestro país.

Entre las limitaciones de nuestro estudio mencionamos que, si bien la muestra fue representativa de la distribución por sexo y edad de la población, no lo fue en materia de distribución por jurisdicciones y nivel de escolaridad, lo cual limita la posibilidad de generalizar los resultados a todos los estratos sociales y a todo el territorio nacional. El sesgo del nivel educativo puede deberse a la distribución y autoadministración del cuestionario a través de Internet, que tiene mayor respuesta entre los grupos con más activos sociales (34). En la gestión de una pandemia como 
la actual, es crucial un estudio más pormenorizado de las percepciones en sectores desfavorecidos de la población, que no han sido abordados en este estudio probablemente por sus limitaciones en el acceso a Internet, por ejemplo, para indagar cómo las carencias habitacionales se vinculan con la posibilidad de prevenir el contagio de coronavirus. Por otro lado, es probable que la percepción de riesgo se modifique en función de las variaciones en la situación epidemiológica de nuestro país y de países cercanos, las medidas gubernamentales que se tomen y la información circulante $(22,24)$. Cabe mencionar que recientemente se ha resaltado que la atención primaria de la salud puede contribuir a la educación en las medidas de higiene y adopción de medidas preventivas (35), con lo cual podría constituirse en un factor clave, considerando que la pandemia tiene impactos desiguales.

Por último, se recomiendan diseños longitudinales o la monitorización periódica de estos indicadores para estudiar los posibles cambios en la percepción del riesgo.

\section{Conclusiones}

Este es el primer estudio en nuestro país en analizar la percepción de riesgo y los factores asociados en esta primera fase de la pandemia, con el espíritu de aportar conocimiento científico que pueda ser utilizado en el diseño de actuaciones preventivas y promover la salud pública. Concluimos que la percepción de riesgo frente al coronavirus en la primera fase de la pandemia en Argentina es moderada. Asimismo, identificamos una mayor percepción de severidad alta en caso de contagio y una mayor percepción de probabilidad de contagio baja. La frecuencia de búsqueda de información y el sentimiento de lejanía del virus son variables que se asocian tanto a la percepción de contagio y como de severidad. La autoeficacia se asoció únicamente con la percepción de severidad.

Los resultados obtenidos destacan la construcción social y emocional de la percepción de riesgo y la importancia de su estudio para el diseño de políticas públicas de prevención y de comunicación sanitaria, en un contexto de emergencia como el actual. Aspectos que deberían considerarse a la hora de diseñar políticas de prevención y abordaje de esta pandemia.

\section{SIN CONFLICTOS DE INTERÉS. SIN FINANCIACIÓN.}

\section{Agradecimientos}

Agradecemos los aportes de Dra. Cecilia Johnson y Dr. Adrián Carbonetti en el diseño del estudio y del cuestionario utilizado. También al personal de apoyo de nuestro lugar de trabajo que colaboró en la difusión del cuestionario. Agradecemos muy especialmente a las personas participantes por su tiempo y contribución. 


\section{Referencios Bibliograficos}

1. Paek HJ y Hove T. Risk perceptions and risk characteristics. En: Oxford Research Encyclopedia of Communication, 2017. https://doi.org/10.1093/acrefore/9780190228613.013.283

2. Conner M. Models of health behaviour. En: Llewellyn C, Ayers S, McManus C et al. Cambridge Handbook of Psychology, health and medicine.1ra ed.Cambridge: Cambridge University Press; 2019. p. 55- 60.

3. Samadipour E, Ghardashi F y Aghaei N. Evaluation of Risk Perception of Covid-19 Disease: A Community-based Participatory Study. Disaster Med Public Health Prep, 2020; 1-20. doi:10.1017/ dmp.2020.311

4. Proske D. Catalogue of risks. Natural, technical, social and health risks. 1ra ed. Berlin: Springer; 2008.

5. Leppin A y Aro A. Risk perceptions related to SARS and avian influenza: theoretical foundations of current empirical research. Int J Behav Med. 2009; 16:7-29.

6. Brown R, Coventry L y Pepper G. COVID-19 risk perceptions and their associations with related media consumption and personal experiences. OSF Preprints, 30 July 2020. Web. https://doi.org/10.31219/osf. io/gcz27

7. Vaughan P. Percepción de los riesgos. En: Organización Mundial de la Salud. Informe sobre la salud en el mundo 2002. Reducir los riesgos y promover una vida sana. 1ra ed. París: Organización Mundial de la Salud; 2002. p. 27-45.https://www.who.int/whr/2002/en/Chapter3S.pdf

8. Alocución de apertura del Director General de la OMS en la rueda de prensa sobre la COVID-19. Organización Mundial de la salud. (Declaración en internet) 2020. 11 de marzo. (Acceso el 15 de abril de 2020) Disponible en: https://www.who.int/es/dg/speeches/detail/who-director-general-s-openingremarks-at-the-media-briefing-on-covid-19---11-march-2020

9. Malecki K, Keating JA y Safdar N. Crisis Communication and public perception of COVID-19 risk in the era of social media. Clin Infect Dis. 2020 Jun 16:ciaa758. doi: 10.1093/cid/ciaa758. Epub ahead of print. PMID: 32544242; PMCID: PMC7337650.

10. Kok G, Jonkers R, Gelissen R, Meertens R, Schaalma H y de Zwart O. Behavioural intentions in response to an influenza pandemic. BMC Publ Health. 2010; 10:174

11. Li, Y., Luan, S., Li, Y., \& Hertwig, R. Changing emotions in the COVID-19 pandemic: A three-wave longitudinal study in the United States and China. Pure MPG. 2020 https://pure.mpg.de/rest/items/ item_3260211/component/file_3260212/content

12. WHO Regional Office for Europe. COVID-19 Snapshot MOnitoring (COSMO): Monitoring knowledge, risk perceptions, preventive behaviours, and public trust in the current coronavirus outbreak. 2020.file://C:/ Users/Lore/Downloads/Guidance\%2520and\%2520protocol.BI\%2520for\%2520COVID-19_160420.pdf

13. McFadden SA, Malik A, Aguolu O, Willebrand K y Omer S. Perceptions of the adult US population regarding the novel coronavirus outbreak. PLoS ONE. 2020; 15(4): e0231808. https://doi.org/10.1371/ journal.pone. 0231808

14. Olapegba Py Ayandele O. Survey data of COVID-19-related knowledge, risk perceptions and precautionary behavior among Nigerians. Data in Brief. 2020, doi: https://doi.org/10.1016/j.dib.2020.105685

15. Motta Zanin G, Gentile E, Parisi A y Spasiano D. A Preliminary Evaluation of the Public Risk Perception Related to the COVID-19 Health Emergency in Italy. Int. J. Environ. Res. Public Health. 2020; 17; 3024.

16. Qian M, Wu Q, Wu P, Hou Z, Liang Y, Cowling B y Yu H. Psychological responses, behavioral changes and public perceptions during the early phase of the COVID-19 outbreak in China: a population based crosssectional survey. MedRxiv. 2020. 02.18.20024448; doi: https://doi.org/10.1101/2020.02.18.20024448

17. Kwok KO, Li KK, Chan HHH, Yi YY, Tang A, Wei WI, et al. Community responses during early phase of COVID-19 epidemic, Hong Kong. Emerg Infect Dis. 2020; 26. https://doi.org/10.3201/eid2607.200500

18. Huynh TLD. The COVID-19 risk perception: A survey on socioeconomics and media attention. Economic Bulletin. 2020; 40 (1): 758-764. 
19. Dryhurst S, Schneider C, Kerr J, Freeman A, Recchia G, van der Bles AM et al. Risk perceptions of COVID-19 around the world. J Risk Res. 2020. DOI: 10.1080/13669877.2020.1758193

20. Ministerio de Salud. Reporte diario 24 de marzo 2020. Accesible en: file://C:/Users/Lore/Downloads/ covid19_informe-diario-matutino-24-03_0\%20(1).pdf Consultado el 25 de mayo de 2020.

21. Sharot T. The optimism bias. Curr Biol. 2011; 21: R941-R945

22. Muñiz C y Corduneanu VI. Percepción de riesgo y consumo mediático durante el inicio de la pandemia de COVID-19 en México. Más poder local. 2020; 41: 44-47

23. Reintjes R, Das E, Klemm C, Richardus JH, Keßler V y Ahmad A. Pandemic Public Health Paradox": Time Series Analysis of the 2009/10 Influenza A/H1N1 epidemiology, media attention, risk perception and public reactions in 5 European countries. PLOS ONE. 2016; 11: e0151258.

24. Walter D, Böhmer MM, Reiter S, Krause G y Wichmann O. Risk perception and information seeking behaviour during the 2009/10 influenza A(H1N1)pdm09 pandemic in Germany. Eurosurveillance. 2012; 17(13): 20131.

25. Sy A y Spinelli H. Dimensiones políticas de una epidemia: el caso de la gripe $\mathrm{A}(\mathrm{H} 1 \mathrm{~N} 1)$ en la prensa escrita de la Argentina. Cad. Saúde Pública 32 (3) 01 Abr 2016 • https://doi.org/10.1590/0102-311X00188414.

26. Ulmer RR, Sellnow TL y Seeger MW. Effective crisis communication: Moving from crisis to opportunity. 4ta ed. Nueva York, Sage Publications; 2017.

27. Slovic P, Finucane ML, Peters E y MacGregor DG. Risk as analysis and risk as feelings: some thoughts about affect, reason, risk, and rationality. Risk Anal. 2004; 24 (2): 311-322.

28. Lupton D. Risk and emotion: towards an alternative theoretical perspective. Health Risk Soc, 2013; 15 : 634-647, DOI: 10.1080/13698575.2013.848847

29. Rimal R y Real K. Perceived risk and efficacy beliefs as motivators of change. Human Commun Res. 2006; 29: 370 - 399.

30. Imes CC, Novosel LM y Burke LE. Heart disease risk and self-efficacy in overweight and obese adults. J Nurse Pract, 2016, 12:710-716.

31. Lima DLF, Dias AA, Rabelo RS, Cruz ID, Costa SC, Nigri FMN y Neri JR. COVID-19 no Estado do Ceará: Comportamentos e crenças na chegada da pandemia. Cien Saude Colet. 2020; 25(5):1575-1586.

32. Jin, JM, Bail P, He W, Wu F, Liu XF, Han DM, et al. Gender differences in patients with COVID-19: focus on severity and mortality. Front Public Health. 2020; 8: 152-157.

33. Marcos-Marcos J, Mateos JT, Gasch-Gallén A y Álvarez-Dardet C. El estudio de la salud de los hombres desde una perspectiva de género: de dónde venimos, hacia dónde vamos.Salud Colect. 2020; 16, e2246.

34. Díaz de Rada V. Ventajas e inconvenientes de la encuesta por Internet. Papers. 2012; 97 (1): 193-223.

35. Vega R. Atención Primaria en salud y COVID-19. Hacia.Promoc Salud. 2020; 25 (2): 17-19 DOI: 10.17151/hpsal.2020.25.2.3 